\title{
Differences of Bone Marrow Features and BCR-ABL Variants in Chronic Granulocytic Leukemia Post Tyrosine Kinase Inhibitor Therapy
}

\author{
Wivina Riza Devi ${ }^{1}, M$ Darwin Prenggono ${ }^{2}$, Purwanto AP $^{3}$, Imam $B^{3}$ \\ ${ }^{1}$ Department of Clinical Pathology, Faculty of Medicine, Lambung Mangkurat University/Ulin General Hospital Banjarmasin, Indonesia. \\ E-mail:wivinarizadevi@gmail.com \\ ${ }^{2}$ Department of Internal Medicine, Faculty of Medicine, Lambung Mangkurat University/Ulin General Hospital Banjarmasin, Indonesia \\ ${ }^{3}$ Department of Clinical Pathology, Faculty of Medicine, Diponegoro University/Dr. Karyadi Hospital Semarang, Indonesia
}

\begin{abstract}
Chronic Granulocytic Leukemia (CGL) occurs due to chromosomal translocation $(9 ; 22)$ known as Philadelphia chromosome. p210 BCR-ABL1 oncogenes are classified into b2a2 and b3a2 transcripts which possibly lead to different clinical manifestations and response to therapy. This study was aimed to prove that there is a difference in bone marrow features and BCR-ABL between remissive and resistant CGL after Tyrosine Kinase Inhibitor (TKI) therapy. This research was an observational study with a cross-sectional design carried out at Ulin Hospital Banjarmasin on 32 subjects. BCR ABL was detected by using PCR and bone marrow features were assessed by using bone marrow aspiration technique. The difference between bone marrow features and BCR-ABL variants was analyzed by using the T-test $(p<0.005)$ and Chi-Square $(p<0.005)$, respectively. There was a difference of $B C R-A B L$ variants with $p=0.091$ and characterized by $M: E$ ratio $(p=0.124)$, myeloblast count $(p=0.063)$, and eosinophil count $(p=0.055)$. Also, there was a difference of bone marrow cellularity $(p=0.000)$ and basophil count $(p=0.016)$ between remissive CGL and resistant CGL patients. There was no difference in BCR $A B L$ variants, myeloblast count and eosinophil count between remissive CGL and resistant CGL patients. However, there was different of bone marrow cellularity and basophil count between remissive CGL and resistant CGL patients.
\end{abstract}

Keywords: Chronic granulocytic leukemia, BCR-ABL variants, bone marrow

\section{INTRODUCTION}

Chronic Granulocytic Leukemia (CGL) is a myeloproliferative disorder of pluripotent hematopoietic stem cell caused by sustained proliferation and dysregulated programmed cell death (apoptosis). Chronic granulocytic leukemia can occur in all ages with predominant cases in adulthood, with $15-20 \%$ adults and $3 \%$ of children with leukemia are diagnosed with CGL. Chronic granulocytic leukemia mostly occurs in the age of 40-60 years with peak incidence age of 53 years. Males more frequently suffer from CGL compared to females with a ratio of $3: 2.30-40 \%$ of CGL patients are asymptomatic. However, clinical manifestations and symptoms of CGL patients are associated with leukocytosis, splenomegaly or anemia. ${ }^{1-3}$

It is estimated that the incidence of $C G L$ with diagnosed, negative, or unknown Ph/BCR-ABL (because no laboratory test is carried out) is approximately $0.8-1.0 / 100.000$ population and becomes a global health problem. The prevalence of
CGL is high and is estimated $15-20 \%$ of all leukemia in developing countries. Geographical and/or ethnicity are known to influence the incidence of CGL. However, the incidence of CGL in Indonesia has not been clearly determined. Lack of data in poor countries leads to an inappropriate report of CGL incidence. $^{1-3}$

Chronic granulocytic leukemia is initially caused by chromosomal translocation 9; 22 which leads to disrupted differentiation of lymphoid and myeloid lineage of hematopoietic stem cell. The prolonged-expression of $B C R-A B L$ in the bone marrow of remissive CGL patients will undergo complete cytogenetic response (CCyR) up to 10 years. Based on the presence of 75 base pairs of e14, p210 BCR-ABL1 oncogenes are classified into b2a2 (e13a2) and b3a2(e14a2) transcript variants; or in some cases, the combination of both. A little is known about the role of the addition of 25 amino acids from b3a2 BCR-ABL oncoproteina 2. It is hypothesized that the addition is related to the immunogenicity of the transcript variants. The 
difference of disease characteristics and prognostic of p210 B transcripts type has not been widely evaluated. ${ }^{4-6}$

The cause of failure to achieve MMR goals in Indonesia has not been identified. However, noncompliance in treatment is suggested to be one of the possible factors. Therefore, it is important to determine the effect of different $B C R-A B L$ gene variants which influence the mechanism of IM in remissive and resistant CGL patients.

\section{METHODS}

This research was an analytical descriptive study with a cross-sectional approach to remissive or resistant CGL patients after therapy. This study was aimed to determine the difference in bone marrow features and $B C R-A B L$ variants in remissive and resistant CGL patients. Samples were obtained from patients in the Hematology Unit or Department of Internal Medicine of Ulin Hospital Banjarmasin, Kalimantan Selatan. Inclusion criteria were CGL adult patients above 18 years old who already received TKI therapy with normal body temperature $\left(36.4^{\circ} \mathrm{C}-37.2^{\circ} \mathrm{C}\right)$ and normal albumin levels. Exclusion criteria were patients with CRP not within the reference range and positive culture, irregular treatment, and non-TKI (Imatinib) treatment. Chronic granulocytic leukemia was diagnosed based on anamnesis, physical examination (observation of splenomegaly) peripheral blood smear, bone marrow aspiration (to determine the phase of $C G L$ ), and positive results of $B C R-A B L$ (b2a2 and $b_{3} 32$ variants of $B C R-A B L$ genes).

The $B C R-A B L$ variants were detected using PCR, while bone marrow features were assessed using bone marrow aspiration by two clinical pathologists. Patients were diagnosed with remissive CGL based on the criteria of Complete Hematologic Response (CHR) characterized by leukocyte count $<10 \times 109 / \mathrm{L}$, no immature granulocyte in peripheral blood smear, basophil count $<5 \%$, thrombocyte count $<450 x$ 109/L, and no splenomegaly observed.

Data were analyzed using SPSS For Release 17.0 computer program tools and descriptive analysis to obtain demographic and subjects characteristics. The characteristics of variables were described by proportions, mean values, Standard Deviations (SD) and minimum-maximum values. The differences in the number of myeloblasts and eosinophils in the bone marrow were analyzed using the T-test on normally distributed data. The differences of $A B R$ $B C R$ variants, bone marrow cellularity, and $M: E$ ratio were analyzed using the Chi-Square test on normally distributed data. The differences of basophil counts in bone marrow were analyzed using the Mann-Whitney test on abnormally distributed data. The significance value of $p<0.005$ was used.

This research was approved by the Health Research Ethics Committee of the Faculty of Medicine, Lambung Mangkurat University with number 574/KEPK-FK UNLAM/EC/XII/2017.

\section{RESULTS AND DISCUSSIONS}

This study involved a total of 34 subjects, with 2 of them were excluded, due to the unwillingness to participate in bone marrow aspiration. Among 32 subjects, there were 14 subjects with b2a2 variant of $B C R-A B L$ and 18 subjects with b3a2 variant of $B C R-A B L$. The subjects were dominated by 25 remissive CGL patients (78\%) followed by 7 resistant CGL patients (22\%). The age range of the subjects was 18 to 60 years, with the length of illness and therapy with Imatinib of 18 to 24 months. A complete description of the variables in this study can be seen in Table 1.

It can be seen from Table 1 that the age range of CGL-diagnosed patients with b2a2 variants was 26-60 years, while b3a2 variants were identified in relatively younger subjects with age of 18-51 years. Likewise, there was a difference of $B C R-A B L$ ratio, with 0.110-100 International scale was reported in b2a2 variants and a relatively lower ratio of 0.0310-57.676 International scale was reported in b3a2 variants of BCR ABL.

The differences of $B C R-A B L$ variants and its effect on the response of therapy with TKI have long been studied; however, it remains controversial to date. From this study involving CGL patients with p210 transcript of BCR-ABL, 18 (53\%) CGL patients with b3a2 variants, 14 (47\%) CGL patients with b2a2 variants of $B C R-A B L$, and no patients with both genes expressions were found (Table 2). It was similar to many previous studies suggesting less frequent expression of b2a2 gene compared to the b3a2 gene, as a study by Al-Achkar et al. which reported $46.7 \%$ b2a2 gene and $51.1 \%$ b3a2 gene in India, $30.2 \%$ b2a2 gene and $67.5 \%$ in Japan, 31\% b2a2 and $61 \%$ b3a2 in Thailand. Contrastingly, similar studies in Argentina reported $41.7 \%$ b2a2 and $37.5 \%$ b3a2 variants, while other studies in Equador found $94.6 \%$ b2a2 and variants $5.4 \%$ b3a2 variants, and a study in the USA reported $48 \%$ b2a2 and $35 \%$ b3a2 variants, with $17 \%$ no BCR-ABL transcription was identified. This difference was possibly due to differences in 
Table 1. Characteristics of CGL patients based on BCR-ABL variants $(n=32)$

\begin{tabular}{lccc}
\hline \multicolumn{1}{c}{ Variable } & b2a2 Variants & b3a2 Variants & P \\
\hline Age (years) & $46(26-60)$ & $34.5(18-51)$ & 0.003 \\
BCR abl I (IS ratio) & $31,405(0.110-100,000)$ & $3.834(0.310-57.676)$ & 0.061 \\
Hb (g/dL) & $10.0(6.8-123)$ & $9.7(5.3-15.5)$ & 0.832 \\
Leukocytes $\left(10^{3} / \mathrm{uL}\right)$ & $210.15(25.8-444.20)$ & $158.1(47.9-396.0)$ & 0.953 \\
Thrombocytes $\left(10^{3} / \mathrm{uL}\right)$ & $348.5(84-878)$ & $498(190-1.544)$ & 0.033 \\
Myeloblast $\%)$ & $3(2-17)$ & $3(1-13)$ & 0.720 \\
Basophils $(\%)$ & $4(1-15)$ & $5(1-11)$ & 0.705 \\
Eosinophils $(\%)$ & $5.5(2-8)$ & $5(2-15)$ & 0.716 \\
Gender & & $11(61.11)$ & 0.480 \\
$\quad$ Male & $9(64.29)$ & $(38.89)$ & \\
Female & $5(35.71)$ & $1.57: 1$ & \\
Male to female ratio & $1.45: 1$ & & 0.287 \\
Phase of CGL & $12(85.71)$ & $16(88.89)$ & \\
Chronic & $2(14.29)$ & $(11.11)$ & 0.439 \\
Accelerated & $9(64.29)$ & $16(88.89)$ & \\
Hematological remission & &
\end{tabular}

Table 2. The differences of $B C R-A B L$ variants between remissive and resistant CGL patients

\begin{tabular}{cccc}
\hline Gene Variants & Remissive n (\%) & Resistant n (\%) & p \\
\hline b2a2 & $9(64.3)$ & $5(35.7)$ & 0.091 \\
b3a2 & $16(88.9)$ & $2(11.1)$ & \\
Total & $25(78)$ & $7(22)$ & \\
\hline
\end{tabular}

sensitivity of the techniques used in the detection of $B C R-A B L$ transcription; however, other researchers suggested that ethnic differences were likely better to determine the different percentage variation of b2a2 and b3a2 genes. ${ }^{7-10}$

In this study, initial routine blood tests before therapy did not show a significant difference between the b2a2 and b3a2 phenotypes. However, the b3a2 phenotype had a relatively higher platelet count compared to b2a2 phenotype with average count of $498\left(190-1,544 \times 10^{3} / \mathrm{uL}\right)$ and 348 $\left(84-878 \times 10^{3} / \mathrm{uL}\right)$ with $p=0.033(\mathrm{p}<0.05)$. The similar results were reported in study Perego et al. that found relatively higher platelet counts of b3a2 phenotype $\left(306 \times 10^{3} / \mathrm{uL}\right)$ compared to those of b2a2 phenotype $\left(616 \times 10^{3} / \mathrm{uL}\right)$ among 88 study subjects, suggesting the differences of characteristics in routine blood tests, especially the number of platelets between CGL patients with $\mathrm{b} 2 \mathrm{a} 2$ and $\mathrm{b} 3 \mathrm{a} 2$ variants of $B C R-A B L$. ${ }^{6,11,12}$

The difference in the $B C R-A B L$ transcripts was presumed to elicit a different response to therapy with TKI. It was similar to the results of a study by Lucas et al. which showed that the b3a2 phenotype had a better response to imatinib compared to the b2a2 phenotype. It was possibly due to the greater activity of tyrosine kinase in the b2a2 phenotype, leading to a need fora higher dose compared to the b3a2 phenotype. Contrastingly, a study by Hanfstein showed no differences in CCyR, MCyR or hematological responses in these two phenotypes, but only differences in platelet counts at the time of diagnosis. From Table 2 it was shown that 9 research subjects (64.3\%) with the b2a2 phenotype underwent $\mathrm{CHR}$, while 16 subjects with b3a2 phenotype had experienced CHR. However, there were no significant differences of the hematological response $(p>0.05)$ between the two phenotypes. Therefore, there might have been no differences in tyrosine kinase transport or activity in the two phenotypes, suggesting no differences in immunological responses or response to Imatinib therapy. ${ }^{12-17}$

Imatinib mesylate is a relatively effective and safe first-line therapy from TKI (target-selective). This therapy inhibits Platelet-Derived Growth Factor (PDGF) and stem cell factor (c-kit) which will cause changes in bone marrow morphology, with the possibility of normal peripheral blood count but abnormal bone marrow features. ${ }^{18-23}$

There are very few literatures and studies that explain the correlation between TKI therapy in CGL 
Table 3. The differences of cellularity and M:E ratio of bone marrow between remissive and resistant CGL patients

\begin{tabular}{|c|c|c|c|}
\hline Variable & Remissive $n(\%)$ & Resistant n (\%) & $\mathbf{p}$ \\
\hline \multicolumn{4}{|l|}{ Cellularity } \\
\hline Hipocellular & $5(20)$ & $0(0)$ & 0.000 \\
\hline Normocellular & $20(80)$ & $2(29)$ & \\
\hline Hipercellular & $0(0)$ & $5(71)$ & \\
\hline \multicolumn{4}{|l|}{ M:E ratio } \\
\hline Decreased & $7(28)$ & 0 & 0.124 \\
\hline Normal & $13(52)$ & $3(43)$ & \\
\hline Increased & $5(20)$ & $4(57)$ & \\
\hline
\end{tabular}

Table 4. The differences in bone marrow features between remissive and resistant CGL patients

\begin{tabular}{ccccc}
\hline Variable & $\mathbf{n}(\%)$ & Mean (SD) & $\mathbf{p}$ & $\mathbf{C l}$ (95\%) \\
\hline Myeloblast & $25(78)$ & $2.5(1.4)$ & 0.063 & $-2.67(-4.31--1.04)$ \\
Remissive & $7(22)$ & $5.2(3.1)$ & & \\
$\quad$ Resistant & & & & \\
Basophil count & $25(78)$ & $1(1-15)$ & 0.016 & \\
$\quad$ Remissive & $7(22)$ & $24(1-11)$ & & $0.713(-2.88-0.03)$ \\
$\quad$ Resistant & & & & \\
Eosinophils & $25(78)$ & $2.7(1.5)$ & 0.055 & \\
$\quad$ Remissive & $7(22)$ & $4.2(2.2)$ & & \\
Resistant & &
\end{tabular}

patients and changes in bone marrow morphology. In a study by Hasserjihan, changes of bone marrow cellularity, M:E ratio, bone marrow fibrosis, and the number of megakaryocytes were found in 53 patients $(50 \%)$ (Table 3 ) with Imanitib therapy, contrast to study by Sunita et al. which showed $54.4 \%$ hypocellularity and $44.1 \%$ normocellularity inbone marrow of CGL patients after TKI therapy. This study found hypocellularity in the bone marrow of 5 remissive CGL patients (20\%) and normocellularity in the bone marrow of 20 remissive CGL patients (80\%). Twenty-nine percent normocellularity, $71 \%$ hypercellularity, and no hypocellularity were found in resistant CGL patients. There were significant differences $(p<0.05)$ of bone marrow cellularity and the basophils count between remissive and resistant CGL patients, but no significant differences were found in the M:E ratio, myeloblast count, and eosinophil count $(p>0.05)$ (Table 4). In a study by Srinivas et al, morphological features of bone marrow were determined based on the cellularity of bone marrow, M:E ratio, dry tap, percentage of the blast, basophil count, and the presence or absence of CCyR-related megakaryocyte abnormalities in CGL patients treated with Imatinib. In addition, according to
May et al, >3-5\% basophil counts in the bone marrow associated with the presence of persistent thrombocytosis indicated the presence of the Philadelphia chromosome that remained positive. Therefore, further studies were still needed to determine the correlation between bone marrow features, cytogenetic responses, and molecular responses by using bone marrow cellularity as the morphological criteria of bone marrow and basophil counts in bone marrow for additional evaluations of Imatinib therapy before obtaining results from assessment of cytogenetic responses and molecularity in CGL patients treated with TKI. ${ }^{24-31}$

Tyrosine kinase inhibitor will bind to the ATP-binding site of the tyrosine kinase protein group including $B C R-A B L$, there by inhibiting tyrosine kinase activity. Tyrosine kinase inhibitor is a targeted therapy that changes the previous CGL therapy in the form of cytotoxic therapy. The use of TKI has a side effect on changes in bone metabolism, supported by the study by Berman et al. which found hypophosphatemia in some CGL patients treated with TKI, associated with phosphaturia and decreased biochemical markers of bone formation and/or resorption (osteocalcin) compared to healthy subjects as control. These findings were also 
associated withan increase of serum parathyroid hormone and 25 hydroxyvitamin D (25-OH D) levels. TKI disrupted bone mineral metabolism by inhibiting PDGF and stem cell factor (c-kit) in monocytes and macrophages, which will cause changes in bone marrow morphology. Osteoblasts are transported from mesenchymal stem cells (MSCs) and its proliferation is inhibited by Imatinib. The direct effect of TKI on osteocytes is still unknown. Tyrosine kinase inhibitor will increase parathyroid hormone (PTH) levels which lead to an increase in RANKL and will subsequently increase the differentiation and activity of osteoclasts. These conditions cause changes in bone metabolism. In this study, bone marrow hypocellularity was observed in 5 subjects with remissive CGL. In the case TKI-treated CGL, it is important to assess bone marrow cellularity within a certain period, because if bone marrow hypocellularityis found, it is necessary to regulate or reduce the dose of therapy for the migrant worker over a period of time. ${ }^{24-29}$

\section{CONCLUSIONS AND SUGGESTIONS}

There were no differences in $B C R-A B L$ variation, $M: E$ ratio, myeloblasts count, and eosinophils count between remissive and resistant CGL patients after treated with TKI. There were differences in bone marrow cellularity and basophils count between remissive and resistant CGL patients after treated with TKI. It was necessary to perform the molecular and cytogenetic responses after therapy to determine the correlation between bone marrow features, and molecular and cytogenetic responses. Therefore, it was expected that bone marrow imaging can help evaluate CGL therapy if molecular and cytogenetic examinations cannot be performed due to limited means.

\section{REFERENCES}

1. Reksodiputro $\mathrm{H}$, Tadjoedin $\mathrm{H}$, Supandiman $\mathrm{I}$, Supandiman I, Acang N, et al. Epidemiology study and mutation profile of patients with chronic myeloid leukemia (LGK) in Indonesian. Journal of Blood Disorders and Transfusion, 2015; 6(3): 1-13.

2. Buyukasik Y, Haznedaroglu IC, Ilhan O. Chronic myeloid leukemia: Practical issues in diagnosis, treatment and follow-up. Int J of Hematology and Oncology, 2010; 20(2): 1-10.

3. Christy. Leukemia mielositik kronik. Dalam: Hematology. $1^{\text {th }}$ Ed., WIMI. 2011; 199-206.

4. Jabbour E, Kantarjian H. Chronic myeloid leukemia: 2016 update on diagnosis, therapy, and monitoring. American Journal of Hematology, 2016; 91(2):
253-255.

5. Kolibaba KS. Molecular monitoring or response in patients with chronic myeloid leukemia. Managed Care. 2013; 40-58.

6. Bilen Y, Erden F. Hematologic, cytogenetic and molecular responses to imatinib therapy for chronic myeloid leukemia: A single-center experience in Turkey. Turk J Med Sci, 2012; 42(1): 31-38.

7. Hasford J, Baccarani M, Hoffmann V. Predicting complete cytogenetic response and subsequent progression-free survival in 2060 patients with LGK on imatinib treatment: the EUTOS score. The American Society of Hematology, 2011; 118(3): 686-690.

8. Usman M, Syed NN, Kakepoto GN, Adil SN, Khursid IA. Chronic phase chronic myeloid leukemia: Response of imatinibmesylate and significance of SOCAL score, age and disease duration in predicting the hematological and cytogenetic response. JAPI, 2007; 56: 103-181.

9. Saußele S, Richter J, Hochhaus A, Mahon F-X. The concept of treatment-free remission in chronic myeloid leukemia. Macmillan Publishers Limited, Leukemia, 2016; 30: 1638-47.

10. Baccarani M, Cortes J, Pane F, Niederwieser D, Saglio G, et al. Chronic myeloid leukemia: An update of concepts and management recommendations of European Leukemia. Net. J of Clin Oncol, 2009; 6041-51.

11. Apperley JF. Part I: Mechanisms of resistance to imatinib in chronic myeloid leukemia. Lancet Oncology, 2007; 8: 1018-29.

12. Liesveld JL, Lichtman MA. Chronic myelogenous leukemia and related disorders. Dalam: William Hematology. $9^{\text {th }}$ Ed., New York, Mc Graw Hill. 2010; 1437-73.

13. Fausel C. Targeted chronic myeloid leukemia therapy: Seeking cure. Supplement to Journal of Managed Care Pharmacy. 2007; 13(8): S8-S12.

14. Wang W, Cortes JE, Tang G, Khoury JD, Bueso Ramos SC, et al. Risk stratification of chromosomal abnormalities in chronic myelogenous leukemia in the era of tyrosine kinase inhibitor therapy. The American Society of Hematology, 2016; 2742-49.

15. Hoffbrand AV, Pettit JE, Vyas Paresh. Chronic myeloid leukemias and myelomonocytic/myeloproliferative disorder. Dalam: Color atlas of clinical hematology. $4^{\text {th }}$ Ed., Philadelpia, Mosby Elsevier, 2009; 233-246.

16. Cortes J, Cardama AQ, Kantarjian HM. Monitoring molecular response in chronic myeloid leukemia. Willey Online Library. PMC 2016 Aug 1. 2010; 1113-20.

17. Hanfstein $B$, Lausaker $M$, Hehlmann R, Saussele $S$, Erben $P$, et al. Distinct characteristics of e13a2 versus e14a2 BCR-ABL, driven chronic myeloid leukemia under first-line therapy with imatinib. Haematologica, 2014; 99(9): 1441-46.

18. Al-Achkar W, Moassass F, Youseff N, Wafa A. Correlation of p210 BCR-ABL transcript variant with clinical, parameters and disease outcome in 45 chronic myeloid leukemia patients. J BUON, 2016; 21(2): $444-449$. 
19. Deb P, Chakrabarti P, Chakrabarty S, Aich R, Nath U, Ray SS, Chaudhuri U. Incidence of BCR-ABL transcript variants in patients with chronic myeloid leukemia: Their correlation with presenting features, risk scores and response to treatment with imatinib mesylate. Indian Journal of Medical and Paediatric Oncology, 2014; 35(1): $26-30$.

20. Hughes TP, Ross DM. Moving treatment-free remission into mainstream clinical practice in CML. The American Society of Hematology, 2016; 128(1): 17-23.

21. Ren R. Mechanisms of BCR-ABL in the pathogenesis of chronic myelogenous leukemia. Nature Reviews Cancer, 2015; 5: $172-180$.

22. Aleman JO, Farooki A, Girotra M. Effects of tyrosine kinase inhibition on bone metabolism: untargeted consequences of targeted therapies. Society for Endocrinology. 2014; R247-R259.

23. Hasserjian R, Boeklin F, Parker S, Chase A, Dhar $S$, et al. ST1571 (imatinib mesylate) reduces bone marrow cellularity and normalizes morphologic features irrespective of cytogenetic response. Hematopathology American Journal of Clinical Pathology, 2012; 117: 360-367.

24. Cotelingan JD. Bone marrow biopsy: Interpretive guidelines for the Surgical Pathologist. Advances in Anatomic Pathology, 2003; 10(1): 8-26.

25. May PC, Khorashod JS, Alitian M, Petotti D, Reid AG. The genetic of chronic myelogemous leukemia in the genetic basis of haematological cancers. $1^{\text {st }}$ Ed.,
London, Willey, 2016; 312-338.

26. Bain BJ, Clark DM, Wilkins BS. The normal bone marrow. Dalam: Bone marrow pathology. $4^{\text {th }}$ Ed., London, Wiley-Blackwell, 2013; 1-51.

27. Lucas CM, Harris RJ, Glannoudis A, Davies A, Knight K, et al. Chronic myeloid leukemia patients with the e13a2 BCR-ABL fusion transcript have inferior response to imatinib compared to patients with the e14a2 transcript. Hematologica Journal, 2009; 94(10): 1362-67.

28. Srinivas BH, Paul TR, Uppin SG, Uppin MS, Jacob RT, Raghunadharao D. Morphologic changes in the bone marrow in patients of Chronic Myeloid Leukemia $(\mathrm{CML})$ treated with imatinib mesylate. Indian $\mathrm{J}$ Hematol Blood Transfus, 2012; 28(3): 162 - 169.

29. Joshi S, Sunita P, Deshmukh C, Gujral S, Amre P, Nair $\mathrm{CN}$. Bone marrow morphological changes in patients of chronic myeloid leukemia treated with imatinib mesylate. Indian Journal of Cancer, 2008; 45(2):45-49.

30. Sunita JS, Joshi S, Desmukh C, Gujral S, Amre P, Nair $\mathrm{CN}$. Bone marrow morphological changes in patients of chronic myeloid leukemia treated with imatinib mesylate. Indian Journal of Cancer, 2008; 45(2): 45-49.

31. Machado MP, Tomaz JP, Metze IL, Souca CA, Vigorito AC, Delamain MT, et al. Monitoring of BCR-ABL levels in chronic myeloid leukemia patients treated with imatinib in the chronic in the chronic phase-the importance of a major molecular response. Rev Bras Hematol Hemoter, 2011; 33(3): 211-5. 